HU-TFT-93-28

\title{
q-Supersymmetric Generalization of von Neumann's Theorem
}

\author{
by \\ M. Chaichian ${ }^{1}$, R. Gonzalez Felipe ${ }^{1,{ }^{*}}$ and P. Prešnajder ${ }^{2,{ }^{* *}}$ \\ ${ }^{1}$ High Energy Physics Laboratory, Department of Physics, P.O. Box 9 \\ (Siltavuorenpenger 20 C), SF-00014 University of Helsinki, Finland \\ ${ }^{2}$ Research Institute for Theoretical Physics, University of Helsinki, P.O. Box 9 \\ (Siltavuorenpenger 20 C), SF-00014 University of Helsinki, Finland
}

May 1993

\begin{abstract}
Assuming that there exist operators which form an irreducible representation of the $q$-superoscillator algebra, it is proved that any two such representations are equivalent, related by a uniquely determined superunitary transformation. This provides with a $q$ supersymmetric generalization of the well-known uniqueness theorem of von Neumann for any finite number of degrees of freedom.
\end{abstract}

\footnotetext{
*) ICSC-World Laboratory; On leave of absence from Grupo de Física Teórica, Instituto de Cibernética, Matemática y Fisica, Academia de Ciencias de Cuba, Calle E No. 309, Vedado, La Habana 4, Cuba.

**) Permanent address: Department of Theoretical Physics, Comenius University, Milynská dolina F2, CS-84215 Bratislava, Slovakia.
} 


\section{Introduction}

In the last few years quantum deformations of Lie groups and Lie algebras have found several applications in mathematics and theoretical physics (see e.g. refs. [1]-[3]). These deformations have been subsequently extended to supergroups and superalgebras [4]-[6]. In particular, the bosonic and fermionic q-oscillators [7], [8], [6] have been used for the realization of different quantum Lie algebras [7]-[9] and quantum superalgebras [6], 10, 11.

A natural question then arises, concerning the relation between different irreducible representations of the $q$-deformed algebras. It is well known that in the case of the classical bosonic and fermionic Heisenberg algebras for harmonic oscillators, this problem is solved by the von Neumann's theorem (see, e.g. [12, [13]), which states that irreducible representations of the bosonic (fermionic) algebra are unitarily equivalent to each other. Similar results also hold for irreducible operator representations of Lie superalgebras [14. Recently, it was proved that the analogue of von Neumann's theorem is also valid in the case of $q$-oscillator algebras [15].

In this letter we shall extend the results of [14] and formulate a quantum supersymmetric generalization of von Neumann's theorem for irreducible representations of $q$-deformed superalgebras. We start from a supercovariant system of $q$-oscillators [11, which are covariant under the coaction of a supergroup, $S U_{q}(n \mid m)$. The latters present the extension of the covariant system of $q$-oscillators proposed in [16, [17]. Assuming suitable domain properties as in [14], we prove that any two representations of the $q$-deformed superalgebra are connected by a unique superunitary transformation. A similar result is also proved to be valid for any finite number, $n$, of bosonic and fermionic independent $q$-oscillators. We present the explicit form of the superunitary transformation operator for the cases $n=1$ and 2.

\section{2 von Neumann's theorem and its extension to $q-$ deformed algebras}

We start by recalling the classical von Neumann's theorem [12, 113. Let $b, b^{+}$and $b^{\prime}, b^{\prime+}$ be two irreducible representations of the Heisenberg algebra,

$$
b b^{+}-b^{+} b=1,
$$

in the Hilbert spaces $H$ and $H^{\prime}$, respectively, and assume that there exist vectors $\mid 0>$ in $H$ and $\mid 0^{\prime}>$ in $H^{\prime}$, such that $b\left|0>=0, b^{\prime}\right| 0^{\prime}>=0$. Then there exists a unitary operator $U$ such that

$$
\begin{aligned}
& b^{\prime}=U b U^{+}, b^{+}=U b^{\prime} U^{+}, \\
& U U^{+}=U^{+} U=1 .
\end{aligned}
$$

A similar theorem also holds in the case of the fermionic algebra [12, [13],

$$
c c^{+}+c^{+} c=1 \text {. }
$$


The above theorem can be formulated in a slightly different form, which we shall utilize further:

Let $b, b^{+}$and $b^{\prime}, b^{\prime+}$ satisfy all the conditions of von Neumann's theorem and let us define the operators $a, a^{+}$and $a^{\prime}, a^{\prime+}$ as

$$
\begin{array}{ll}
a=\varphi(N) b & , \quad a^{+}=b^{+} \varphi^{+}(N), \\
a^{\prime}=\varphi\left(N^{\prime}\right) b^{\prime} & , \quad a^{\prime+}=b^{\prime+} \varphi^{+}\left(N^{\prime}\right)
\end{array}
$$

where

$$
N=b^{+} b, N^{\prime}=b^{+} b^{\prime},
$$

are the number operators and $\varphi$ is a well-behaved function.

Then for the representations $a, a^{+}$and $a^{\prime}, a^{\prime+}$ in some Hilbert (sub)spaces $H_{a}$ and $H_{a}^{\prime}\left(H_{a} \subseteq H, H_{a}^{\prime} \subseteq H^{\prime}\right)$, respectively, von Neumann's theorem also holds, i.e., $a, a^{+}$and $a^{\prime}, a^{\prime+}$ are irreducible representations, there exist vectors $\mid 0>$ in $H_{a}$ and $\mid 0^{\prime}>$ in $H_{a}^{\prime}$ such that $a\left|0>=0, a^{\prime}\right| 0^{\prime}>=0$ and there exists a unitary operator $U$ such that

$$
a^{\prime}=U a U^{+}, a^{+}=U a^{+} U^{+}, N^{\prime}=U N U^{+} .
$$

To prove (6) we notice that since $b, b^{+}$and $b^{\prime}, b^{\prime+}$ satisfy the conditions of von Neumann's theorem, it follows that there exists a unitary operator $U$ such that relations (2) hold. From (2) and (5) it follows that

$$
N^{\prime}=b^{\prime+} b^{\prime}=U b^{+} U^{+} U b U^{+}=U N U^{+},
$$

and the same relation is also valid for the function $\varphi(N)$,

$$
\varphi\left(N^{\prime}\right)=U \varphi(N) U^{+} .
$$

Now, from the definition of the operators $a, a^{+}$(eqs. (4)) we have

$$
U a U^{+}=U \varphi(N) b U^{+}=U \varphi(N) U^{+} U b U^{+}=\varphi\left(N^{\prime}\right) b^{\prime}=a^{\prime},
$$

and, similarly,

$$
U a^{+} U^{+}=a^{\prime+}
$$

Relations (6) are thus proved.

It is also clear that in the spaces $H_{a}$ and $H_{a}^{\prime}$ there exist vectors $\mid 0>$ and $\mid 0^{\prime}>$ such that $a\left|0>=0, a^{\prime}\right| 0^{\prime}>=0$. Finally, $a, a^{+}$and $a^{\prime}, a^{\prime+}$ are two irreducible representations in the Hilbert spaces $H_{a}, H_{a}^{\prime}$, respectively. This follows from the definitions (4) and the fact that $b, b^{+}$and $b^{\prime}, b^{\prime+}$ are irreducible representations in $H, H^{\prime}$.

The same conclusion also holds obviously for the case of fermionic oscillators.

From the above theorem, it follows, in particular, that any two irreducible representations of the $q$-deformed bosonic oscillator algebra [7], [8], [6]

$$
\begin{aligned}
& a a^{+}-q a^{+} a=q^{-N}, \\
& {[N, a]=-a,\left[N, a^{+}\right]=a^{+},}
\end{aligned}
$$

are connected by a unique unitary transformation. Indeed, by taking $\varphi(N)=\sqrt{\frac{[N+1]}{N+1}}$ in (4), where $[n]=\frac{q^{n}-q^{-n}}{q-q^{-1}}$, we obtain the $q$-oscillator algebra (8), provided that $a^{+} a=$ 
$[N], a a^{+}=[N+1]$, i.e. in the Fock space of (8) [18]. For the exceptional values of $q$ being the $m$-th root of unity, $q=e^{ \pm i \pi / m},[m]=0$ and thus $\varphi(m-1)=0$. In such a case the Hilbert space $H_{a}$ becomes finite, $m$-dimensional. A similar statement is also valid in the case of the $q$-deformed fermionic algebra [6]

$$
\begin{aligned}
& f f^{+}+q f^{+} f=q^{M}, \\
& {[M, f]=-f,\left[M, f^{+}\right]=f^{+}}
\end{aligned}
$$

which can be obtained from the algebra (3) by means of the change of operators

$$
f=q^{\frac{M}{2}} c, f^{+}=c^{+} q^{\frac{M}{2}} .
$$

Let us remark that the above statements were proved in ref. [15] by following a proof in the same line as the original von Neumann's theorem for usual (nondeformed) oscillators and valid also for generic values of $q$ including the exceptional values of $m$-th root of unity. The same theorem is also valid in the case of the other $q$-fermionic algebra [19] given by the commutation relations

$$
\begin{gathered}
f f^{+}+q f^{+} f=q^{-M}, \\
{[M, f]=-f,\left[M, f^{+}\right]=f^{+} .}
\end{gathered}
$$

In fact, we can obtain the latters from the bosonic algebra (1) by means of the transformation,

$$
f=\sqrt{\frac{[M+1]^{f}}{M+1}} b, f^{+}=b^{+} \sqrt{\frac{[M+1]^{f}}{M+1}},
$$

where $[n]^{f}=\frac{q^{-n}-(-1)^{n} q^{n}}{q+q^{-1}}$, since in the Fock space we have the relations $f^{+} f=[M]^{f}, f f^{+}=$ $[M+1]^{f}$.

\section{3 q-Supersymmetric von Neumann's Theorem}

In this section we formulate our main result, namely, we prove that an irreducible representation of the $q$-deformed superalgebras is, up to a superunitary transformation, unique, in the following sense:

Theorem (i)

Let $Z=\left\{B, B^{+}, F, F^{+}\right\}$be an irreducible operator family, which satisfies the $q$ deformed superalgebra [11]:

$$
\begin{gathered}
B F=q F B, B^{+} F^{+}=q^{-1} F^{+} B^{+}, \\
B F^{+}=q^{-1} F^{+} B, B^{+} F=q F B^{+}, \\
F^{2}=\left(F^{+}\right)^{2}=0, \\
B B^{+}-q^{-2} B^{+} B=1+\left(q^{-2}-1\right) F^{+} F, \\
F F^{+}+F^{+} F=1 ;
\end{gathered}
$$


where $q$ is a real number, $F, F^{+}$are bounded operators on the separable Hilbert space $H$ and $B, B^{+}$are densely defined closed operators in $H$. Let $\theta$ be a Grassmann variable, such that

$$
\begin{aligned}
\{\theta, F\} & =\left\{\theta, F^{+}\right\}=\theta^{2}=0, \\
{[\theta, B] } & =\left[\theta, B^{+}\right]=0 .
\end{aligned}
$$

Let $D$ and $G$ be densely defined closed linear operators and define on a suitable domain 114

$$
\begin{gathered}
B^{\prime}=B+\theta D, \quad B^{++}=B^{+}-\theta D^{+}, \\
F^{\prime}=F+\theta G, \quad F^{++}=F^{+}+\theta G^{+}, \\
G=G_{00}\left(B, B^{+}\right)+G_{11}\left(B, B^{+}\right) F^{+} F, \\
D=D_{10}\left(B, B^{+}\right) F^{+}+D_{01}\left(B, B^{+}\right) F,
\end{gathered}
$$

where $G$ and $D$ are assumed to be even and odd Grassmann elements, respectively. Assume that the operator family $Z^{\prime}=\left\{B^{\prime}, B^{\prime+}, F^{\prime}, F^{\prime+}\right\}$ also fulfills the algebra (10) on a suitable domain of definition.

Then, under the above conditions, there exists a uniquely determined self-adjoint odd operator $A$, such that

$$
\begin{gathered}
G=\{A, F\}, D=[A, B], \\
A=F^{+} G_{00}\left(B, B^{+}\right)+G_{00}^{+}\left(B, B^{+}\right) F=A^{+},
\end{gathered}
$$

and the transformation (12) is implemented by the superunitary operator $e^{\theta A}$ such that

$$
\begin{gathered}
e^{\theta A} B e^{-\theta A}=B+\theta[A, B]=B^{\prime}, \\
e^{\theta A} F e^{-\theta A}=F+\theta\{A, F\}=F^{\prime} .
\end{gathered}
$$

Under the conditions of the above theorem, we have

$$
\begin{gathered}
G_{11}\left(B, B^{+}\right)=G_{00}\left(q B, q B^{+}\right)-G_{00}\left(B, B^{+}\right) \\
D_{10}\left(B, B^{+}\right)=q G_{00}\left(q B, q B^{+}\right) B-B G_{00}\left(q B, q B^{+}\right), \\
D_{01}\left(B, B^{+}\right)=q^{-1} G_{00}^{+}\left(B, B^{+}\right) B-B G_{00}^{+}\left(B, B^{+}\right) .
\end{gathered}
$$

The transformation $e^{\theta A}=1+\theta A$ is called superunitary if $A$ is odd and self-adjoint. In particular, from this definition it follows that $\{\theta, A\}=0$ and the latter is fulfilled only when $\theta$ satisfies the commutation relations (11). 


\section{Remark:}

The Grassmann variable $\theta$, being on the $q$-plane of the oscillators given in (10), can in general have the $q$-commutation relations with the elements of the algebra as:

$$
\begin{array}{ll}
\theta_{q} F+p F \theta_{q}=0, & \theta_{q} F^{+}+p^{-1} F^{+} \theta_{q}=0, \\
\theta_{q} B-r B \theta_{q}=0, & \theta_{q} B^{+}-r^{-1} B^{+} \theta_{q}=0,
\end{array}
$$

with $p$ and $r$ real numbers . If however, we use the above $q$-commutation relations with $p$ and $r$, instead of commutation relations (11), from the requirement of oddness of operator $A$ and the superunitarity condition $\left(e^{\theta_{q} A}\right)^{+}=e^{-\theta_{q} A}$, i.e. $A^{+} \theta_{q}=-\theta_{q} A$, one arrives at the following " $q$-self adjointness" condition,

$$
A^{+}\left(B, B^{+}, F, F^{+}\right)=A\left(r B, r^{-1} B^{+}, p F, p^{-1} F^{+}\right),
$$

instead of the usual one. The latter restriction seems rather unnatural, since a usual self-adjoint operator $A$ on the original algebra of oscillators (10) now acquires restrictions by the inclusion of an additional auxiliary Grassmann element $\theta_{q}$. Thus for our purpose we can restrict ourselves to the case $p=r=1$, i.e., to the commutation relations (11).

\section{Proof of the theorem:}

The proof is actually quite similar to the one given in [14 for the classical Lie superalgebras.

From the relations $F^{2}=F^{\prime 2}=0$ and using (11a) we have, according to (12), $F^{\prime 2}=$ $\theta[G, F]=0$, from which we obtain

$$
[G, F]=0 .
$$

Relations (10a), (10b) imply that for any function $g\left(B, B^{+}\right)$it holds

$$
\begin{aligned}
& g\left(B, B^{+}\right) F=F g\left(q B, q B^{+}\right), \\
& g\left(B, B^{+}\right) F^{+}=F^{+} g\left(q^{-1} B, q^{-1} B^{+}\right) .
\end{aligned}
$$

With the ansatz (13) and using (10e) and (19) we find

$$
[G, F]=\left\{G_{00}\left(B, B^{+}\right)-G_{00}\left(q^{-1} B, q^{-1} B^{+}\right)-G_{11}\left(q^{-1} B, q^{-1} B^{+}\right)\right\} F .
$$

Comparing (18) and (20) we get

$$
G_{11}\left(B, B^{+}\right)=G_{00}\left(q B, q B^{+}\right)-G_{00}\left(B, B^{+}\right)
$$

*These $q$-commutation relations, because of associativity, are of course compatible with the supersymmetric $q$-Jacobi identities

$$
\begin{gathered}
{\left[[A, B\}_{\left(q_{3}, q_{3}^{-1}\right)}, C\right\}_{\left(\frac{q_{1}}{q_{2}}, q_{2}\right.}+(-1)^{\eta_{A}\left(\eta_{B}+\eta_{C}\right)}\left[[B, C\}_{\left(q_{1}, q_{1}^{-1}\right)}, A\right\}_{\left(\frac{q_{2}}{q_{3}}, q_{q_{3}}\right)}} \\
+(-1)^{\eta_{C}\left(\eta_{A}+\eta_{B}\right)}\left[[C, A\}_{\left(q_{2}, q_{2}^{-1}\right)}, B\right\}_{\left(\frac{q_{3}}{q_{1}}, \frac{q_{1}}{q_{3}}\right)}=0,
\end{gathered}
$$

where $\eta_{Z}=1$ if $Z$ is odd, $\eta_{Z}=0$ if $Z$ is even and $[A, B\}_{(p, q)} \equiv p A B \mp q B A$, where we take the plus sign when both $A$ and $B$ are odd, otherwise we take the minus sign. The above expression represents the most general form of the supersymmetric $q$-Jacobi identities, which includes three arbitrary complex parameters $q_{1}, q_{2}$ and $q_{3}$. 
and substituting it into (13) we obtain

$$
G=G_{00}\left(B, B^{+}\right) F F^{+}+G_{00}\left(q B, q B^{+}\right) F^{+} F .
$$

Let us now take $G$, in the required form (14), as

$$
G=\{A, F\},
$$

where $A$ is odd. Assuming that $A$ is self-adjoint, i.e. $A^{+}=A$, we can write

$$
A=F^{+} \alpha\left(B, B^{+}\right)+\alpha^{+}\left(B, B^{+}\right) F
$$

and we have from (23),

$$
G=\alpha\left(q B, q B^{+}\right) F^{+} F+\alpha\left(B, B^{+}\right) F F^{+}
$$

Comparing (22) and (25), we find

$$
\alpha\left(B, B^{+}\right)=G_{00}\left(B, B^{+}\right),
$$

and by substituting it back into (24), the relation (15) is obtained.

Now to find $D$, we use relations (10a) and (11). We have

$$
B^{\prime} F^{\prime}-q F^{\prime} B^{\prime}=\theta\{D F+B G-q G B+q F D\}=0 .
$$

Substituting (23) into (26) and using (10a), we will obtain then that

$$
\{D-[A, B]\} F+q F\{D-[A, B]\}=0 .
$$

Similarly, from (10b), (11) and $G^{+}=\left\{A, F^{+}\right\}$we obtain

$$
\{D-[A, B]\} F^{+}+q^{-1} F^{+}\{D-[A, B]\}=0 .
$$

Thus, from eqs. (27) we conclude that

$$
D=[A, B] .
$$

Finally, the operator $e^{\theta A}$ is superunitary since $A$ is odd and self-adjoint (see eq. (24)). Moreover, we have

$$
\begin{gathered}
e^{\theta A} B e^{-\theta A}=(1+\theta A) B(1-\theta A)=B+\theta[A, B]=B^{\prime}, \\
e^{\theta A} F e^{-\theta A}=(1+\theta A) F(1-\theta A)=F+\theta\{A, F\}=F^{\prime},
\end{gathered}
$$

i.e. relations (16) hold. The proof of the relations (17) is straighforward: eq. (17a) was already proved (see eq. (21)) and relations (17b) follow from (13), (14), (15) and (19). This completes the proof. When $q=1$, we reproduce the results of [14].

Let us remark that a pair of one bosonic, $b, b^{+}$, and one fermionic, $f, f^{+}$, independent $q$-oscillators (which satisfy the relations (30)), can also be introduced by means of the transformation [11]

$$
\begin{array}{lll}
b=q^{\frac{N}{2}+M} B & , \quad f=q^{\frac{M}{2}} F, \\
{[N, B]=-B} & , \quad\left[N, B^{+}\right]=B^{+}, N^{+}=N, \\
{[M, F]=-F} & , \quad\left[M, F^{+}\right]=F^{+}, M^{+}=M,
\end{array}
$$


where $B$ and $F$ are the elements of supercovariant algebra (10). In this case, the uniqueness (up to a superunitary transformation) of any irreducible representation of $b, b^{+}, N, f, f^{+}, M$ is given by the following theorem.

\section{Theorem (ii)}

Let $Z=\left\{b, b^{+}, N, f, f^{+}, M\right\}$ be an irreducible operator family, which satisfies the $q$-oscillator algebra

$$
\begin{gathered}
{[b, f]=0,\left[b, f^{+}\right]=0,} \\
f^{2}=\left(f^{+}\right)^{2}=0, \\
b b^{+}-q^{-1} b^{+} b=q^{N}, \\
f f^{+}+q f^{+} f=q^{M}, \\
{[N, b]=-b,\left[N, b^{+}\right]=b^{+},} \\
{[M, f]=-f,\left[M, f^{+}\right]=f^{+},}
\end{gathered}
$$

on a suitable domain of definition; $q$ is real. Let $\theta$ be a Grassmann variable,

$$
\begin{aligned}
& \{\theta, f\}=\left\{\theta, f^{+}\right\}=\theta^{2}=0, \\
& {[\theta, b]=\left[\theta, b^{+}\right]=0 .}
\end{aligned}
$$

Define

$$
\begin{gathered}
b^{\prime}=b+\theta D, \quad f^{\prime}=f+\theta G, \\
D=D_{10}\left(b, b^{+}, M\right) f^{+}+D_{01}\left(b, b^{+}, M\right) f, \\
G=G_{00}\left(b, b^{+}, M\right)+G_{11}\left(b, b^{+}, M\right) f^{+} f,
\end{gathered}
$$

where $D$ and $G$ are the even and odd Grassmann elements, respectively, and have the most general form as in (32). Assume that the operator family $Z^{\prime}=\left\{b^{\prime}, b^{\prime+}, N^{\prime}, f^{\prime}, f^{\prime+}, M^{\prime}\right\}$ also fulfills the algebra (30) on a suitable domain of definition.

Then there exists a uniquely determined self-adjoint odd operator $A$, such that one can write

$$
\begin{gathered}
G=\{A, f\}, \quad D=[A, b], \\
A=f^{+} \alpha\left(b, b^{+}, M\right)+\alpha^{+}\left(b, b^{+}, M\right) f=A^{+}
\end{gathered}
$$

and the transformation (32) is implemented by the superunitary operator $e^{\theta A}$ such that

$$
\begin{gathered}
e^{\theta A} b e^{-\theta A}=b^{\prime}, \\
e^{\theta A} f e^{-\theta A}=f^{\prime} .
\end{gathered}
$$

Under the conditions of the above Theorem (ii), we have

$$
G_{00}\left(b, b^{+}, M\right)=\alpha\left(b, b^{+}, M\right) q^{M}, G_{11}\left(b, b^{+}, M\right)=\alpha\left(b, b^{+}, M-1\right)-q \alpha\left(b, b^{+}, M\right)
$$

and

$$
D_{10}\left(b, b^{+}, M\right)=\left[\alpha\left(b, b^{+}, M-1\right), b\right], D_{01}=\left[\alpha^{+}\left(b, b^{+}, M\right), b\right] .
$$

The proof is similar to the one of Theorem (i) and will not be given. 
Let us notice that from the Theorem (ii), it also follows that

$$
\begin{gathered}
e^{\theta A} N e^{-\theta A}=N+\theta[A, N]=N^{\prime}, \\
e^{\theta A} M e^{-\theta A}=M+\theta[A, M]=M^{\prime} .
\end{gathered}
$$

Indeed, relations (35) imply that for any functions $\varphi\left(b b^{+}, b^{+} b\right)$ and $\psi\left(f f^{+}, f^{+} f\right)$, one has

$$
\begin{aligned}
e^{\theta A} \varphi\left(b b^{+}, b^{+} b\right) e^{-\theta A} & =\varphi\left(b^{\prime} b^{++}, b^{++} b^{\prime}\right), \\
e^{\theta A} \psi\left(f f^{+}, f^{+} f\right) e^{-\theta A} & =\psi\left(f^{\prime} f^{\prime+}, f^{\prime+} f^{\prime}\right) ;
\end{aligned}
$$

$\varphi=N$ and $\psi=M$ are just particular cases of these functions (see eqs. (30c) and (30d) respectively).

Here we would like to mention that there exists a relation between the transformations of Theorem (i) and of Theorem (ii). Indeed, if one takes in (34)

$$
\alpha\left(b, b^{+}, M\right)=q^{-\frac{M}{2}} G_{00}\left(B, B^{+}\right)
$$

where $B=q^{-\frac{N}{2}-M} b, F=q^{-\frac{M}{2}} f$ according to (29), it is straighforward to show that the superunitary transformation generated by (34), (35) with $\alpha$ given in (38), corresponds to the superunitary transformation (15), (16) of Theorem (i), and therefore eqs. (17) are satisfied.

Also it will be interesting to find a direct relation between the superunitary transformations of the usual (undeformed) and of the $q$-deformed cases obtained here, in the same way as such a relation exists for the nonsupersymmetric $q$-oscillators treated in section 2 .

To summarize, we have shown that the irreducible representations of the $q$-superoscillator algebra are equivalent and are related by a unique superunitary transformation. The $q-$ supersymmetric generalization of von Neumann's theorem, presented above, is $(N=1$ supersymmetry) for only one bosonic and one fermionic degrees of freedom. Our results can be extended to the supersymmetric case with any number of bosonic and fermionic degrees of freedom. The theorem now can be formulated as follows:

Theorem (finite degrees of freedom):

Let $Z=\left\{b_{k}, b_{k}^{+}, N_{k}, f_{k}, f_{k}^{+}, M_{k} ; k=1, \ldots, n\right\}$ be an irreducible operator set, which satisfies the $q$-oscillator algebra

$$
\begin{gathered}
b_{k} b_{k}^{+}-q^{-1} b_{k}^{+} b_{k}=q^{N_{k}}, \\
f_{k} f_{k}^{+}+q f_{k}^{+} f_{k}=q^{M_{k}}, \\
{\left[N_{k}, b_{k}\right]=-b_{k},\left[N_{k}, b_{k}^{+}\right]=b_{k}^{+},} \\
{\left[M_{k}, f_{k}\right]=-f_{k},\left[M_{k}, f_{k}^{+}\right]=f_{k}^{+},}
\end{gathered}
$$

with all the other (anti)commutation relations vanishing (i.e., independent system of $q$ oscillators); $q$ is real.

Assume now that another set of operators $Z^{\prime}=\left\{b_{k}^{\prime}, b_{k}^{\prime+}, N_{k}^{\prime}, f_{k}^{\prime}, f_{k}^{\prime+}, M_{k}^{\prime} ; k=1, \ldots n\right\}$ also satisfies the same algebra (39). Then the two sets $Z$ and $Z^{\prime}$ are equivalent, related by a unique superunitary transformation such that

$$
\begin{aligned}
& b_{k}^{\prime}=U b_{k} U^{+}, \quad f_{k}^{\prime}=U f_{k} U^{+} \\
& U=e^{\theta A}
\end{aligned}
$$


with the same relation between the remaining elements of the two sets. In (40) $\theta$ is a Grassmann variable satisfying the (anti)commutation relations (31) for all the $b_{k}, b_{k}^{+}, f_{k}, f_{k}^{+}$and $A$ is a self-adjoint odd operator.

The proof of the theorem can be most easily performed by the method of induction in the number of degrees of freedom, $n$. In addition to the explicit form of the superunitary operator for $n=1$ given in Theorem (ii), we present below explicitly the formulae for the case $n=2$.

Define

$$
b_{k}^{\prime}=b_{k}+\theta D_{k}, f_{k}^{\prime}=f_{k}+\theta G_{k}, \quad k=1,2,
$$

where $G_{k}$ have the most general form

$$
\begin{gathered}
G_{k}=G_{k}^{0}+G_{k}^{1} f_{1}^{+} f_{1}+G_{k}^{2} f_{2}^{+} f_{2}+G_{k}^{12} f_{1}^{+} f_{1} f_{2}^{+} f_{2} \\
+H_{k} f_{1} f_{2}+H_{k}^{\prime} f_{1}^{+} f_{2}^{+}+K_{k} f_{1} f_{2}^{+}+K_{k}^{\prime} f_{1}^{+} f_{2}
\end{gathered}
$$

all the coefficients in (42) can depend on $b_{\ell}, b_{\ell}^{+}, N_{\ell}, M_{\ell}(\ell=1,2)$.

From the anticommutation relations $\left\{f_{k}^{\prime}, f_{\ell}^{\prime}\right\}=\left\{f_{k}^{\prime+}, f_{\ell}^{\prime+}\right\}=\left\{f_{1}^{\prime}, f_{2}^{\prime+}\right\}=\left\{f_{1}^{\prime+}, f_{2}^{\prime}\right\}=0$, we obtain that $H_{k}^{\prime}=K_{k}^{\prime}=0$ and that the coefficients $G_{k}^{k}, G_{k}^{12}, H_{k}, K_{k}(k=1,2)$ can be expressed in terms of $G_{1}^{0}, G_{2}^{0}, G_{2}^{1}, G_{1}^{2}$ and their hermitian conjugates. Now if we write $G_{k}$ in the form $G_{k}=\left\{A, f_{k}\right\}$, then it is straightforward to show that there exists a unique self-adjoint odd operator $A$ given by

$$
A=\sum_{k} f_{k}^{+} G_{k}^{0} q^{-M_{k}}+\sum_{k \neq \ell} f_{k}^{+} f_{\ell}^{+} f_{\ell} G_{k}^{\ell} q^{-M_{k}}+\text { h.c. }
$$

and from the commutativity between the $b_{k}^{\prime}$ and $f_{\ell}^{\prime}$, we obtain the required form $D_{k}=$ $\left[A, b_{k}\right]$.

\section{Acknowledgements}

It is our pleasure to thank A. Demichev for useful discussions and several clarifying remarks. R.G.F. would like to thank ICSC-World Laboratory for financial support. P.P. is grateful to the Research Institute for Theoretical Physics, University of Helsinki, for the hospitality.

\section{References}

[1] L.D. Faddeev, N.Y. Reshetikhin and L.A. Takhtajan, Algebra i Analys 1 (1989) 178.

[2] Proceedings of the First Euler International Mathematical Institute Workshop on Quantum Groups, Oct. - Dec. 1990, P. Kulish, ed. (Springer, Berlin, 1991). 
[3] Proceedings of XXI Conference on Differential Geometric Methods in Theoretical Physics, Tianjin, China, June 1992 (World Scientific, to appear).

[4] Yu. I. Manin, Commun. Math. Phys. 123 (1989) 163.

[5] R. Kulish and N. Reshetikhin, Lett. Math. Phys. 18 (1989) 143.

[6] M. Chaichian and P. Kulish, Phys. Lett. B 234 (1990) 72.

[7] A.J. Macfarlane, J. Phys. A: Math. Gen. 22 (1989) 4581.

[8] L.C. Biedenharn, J. Phys. A: Math. Gen. 22 (1989) L 873.

[9] T. Hayashi, Commun. Math. Phys. 127 (1990) 129.

[10] M. Chaichian, P. Kulish and J. Lukierski, Phys. Lett. B 237 (1990) 401.

[11] M. Chaichian, P. Kulish and J. Lukierski, Phys. Lett. B 262 (1991) 43.

[12] F.A. Berezin, The method of second quantization (Academic Press, New York and London, 1966).

[13] C.R. Putnam, Commutation properties of Hilbert space operators and related topics (Springer, Berlin, 1967).

[14] H. Grosse and L. Pittner, J. Math. Phys. 29 (1988) 110.

[15] M. Chaichian, M.N. Mnatsakanova and Y.S. Vernov, Preprint Univ. of Helsinki, HUTFT-93-6 (1993).

[16] W. Pusz and S.L. Woronowicz, Rep. Math. Phys. 27 (1989) 231.

[17] W. Pusz, Rep. Math. Phys. 27 (1989) 349.

[18] P. Kulish and E. Damaskinsky, J. Phys. A 23 (1990) L 415.

[19] R. Parthasarathy and K. S. Viswanathan, J. Phys. A: Math. Gen. 24 (1991) 613; J. Beckers and N. Debergh, J. Phys. A: Math. Gen. 24 (1991) L 1277. 